\title{
AN OUTBREAK OF LEGIONNAIRES' DISEASE AT HEYSHAM POWER STATION, 1981
}

\section{FROM THE ARCHIVES}

Last year's outbreak of a Legionella infection in Barrow has revived memories of that in the Phase 1 construction site of Heysham Power Station during September and October 1981.

Whilst there were only a few cases, it was important, as little was known about the organism at that time, and it was not only the first outbreak in the country in an industrial setting, but also one of the few where aerosol spread from a cooling tower was convincingly demonstrated.

\section{ONSET}

The Phase 1 site had been under construction since 1971. At the time of the outbreak, there were about 2,500 workers there, employed by 47 sub-contractors. A similar number were employed on the adjacent Phase 2 site, which was at a less advanced stage of construction.

The first patient to come to the attention of John Dyer, the district medical officer, had fallen ill whilst on holiday in Scotland and had been admitted to the City Hospital in Edinburgh with a diagnosis of Legionella pneumonia. Enquiries at this stage revealed that he had attended the medical centre the day before his holiday with a pyrexia of $39.4^{\circ} \mathrm{C}$. He had been deployed at many different parts of the site over the previous month, mostly outside the main buildings, but had not used the showers or baths. Dr Dyer discussed the situation with a doctor at the Department of Health in London, when it was agreed that as it appeared to be an isolated case, with no obvious cause of origin, there were insufficient grounds for a detailed hunt for the source, which might attract alarmist publicity. Two weeks later, a consultant chest physician reported that two other men employed at the power station had been admitted to the Royal Lancaster Infirmary with pneumonia, with serology indicating Legionella infection. One of these patients had a colleague who had recently died of pneumonia whilst on holiday and was subsequently cremated.

Thus there was strong evidence that there had indeed been a source of infection at Phase 1 during the preceding month or so. On the advice of an epidemiologist at the Communicable Diseases Surveillance Centre in London the following steps were instigated:

- case finding

- case control study

- environmental survey.

\section{CASE FINDING}

This was aimed at detecting all individuals either on site or in the community who had had recent pneumonia or fever with respiratory illness. There was a review of sickness certification of site employees and attendance at the site medical centres from the beginning of September. This was repeated for the Phase 2 site.

Hospital staff and GPs in the Lancaster and surrounding districts were contacted with the request to review all patients with the above illnesses since 1st September. In addition, local hospital inpatient records were reviewed and people with possibly related illnesses were followed up, either as outpatients or via their GPs. Where possible, the sera of all such patients were checked for antibody to Legionella pneumonia.

Three employees were identified as having had pneumonia. Two were treated by their GPs and the other continued at work. All three gave high antibody titres. Of the 79 people ( $32 \%$ of the workforce) who had reported to the medical centre or submitted sick notes, 11 had various levels of antibody to four sera groups.

A case-searching exercise at the Phase 2 site did not reveal any serological evidence of exposure to Legionella amongst those having had recent respiratory tract or 'flu-like' illnesses ( 85 people, $4.3 \%$ of the workforce).

\section{CASE CONTROL STUDY}

The purpose of this was to gather evidence of exposure to Legionella in Phase 1 and to compare it with a control random sample.

As it was apparent that the Phase 2 workforce - entirely distinct from Phase 1 - was unaffected by this outbreak, it afforded an ideal population with which to match the Phase 1 workers. Both sites had similar numbers of employees drawn from a wide area, and the other factors such as occupation, age, sex, social grouping and lifestyle were presumably alike.

A random systemic sample of 100 men (1 in 25 on the payroll) from Phase 1 was invited to give a blood sample for serology and to answer a detailed questionnaire in an interview; 93 accepted. This included details of areas on the site where they had worked, visited, washed etc, travel, smoking and recent illness. Ten had low antibody titres.

A similar review was carried out on Phase 2 where again 100 men were randomly selected and invited to participate; 79 accepted. Blood was taken and an appropriate questionnaire administered. None was found to have measurable Legionella antibodies. 


\section{ENVIRONMENTAL SURVEY}

As soon as the first three cases were reported, water samples were taken from virtually every water outlet on the site, including hot and cold taps, showers, drinking fountains and fire sprinkler systems, for examination and culture at the Preston Public Health Laboratory. No Legionella was isolated. Because of the huge number of water outlets and lack of evidence as to the likely source of the infection, the samples were fairly small.

Similarly, samples were taken from Phase 2 outlets. Following sampling on the Phase 1 site, cleaning measures were carried out on all shower units, toilets and water tanks, and these were treated with chlorination at five parts per million. All showerheads were replaced and the shower units treated with disinfectant. Hot water temperatures were raised to $60^{\circ} \mathrm{C}$ to impede the growth of the organism, if present.

Data from the local meteorological station were examined, particularly in respect of wind direction and speed and maximum and minimum temperatures. The wind directions were grouped by quadrants and in both the preceding months the greatest proportion of readings showed the wind coming from the southwest quadrant, $55 \%$ and $47 \%$ respectively.

\section{RESULTS}

Histories of working in or visiting work areas on the site were compared for cases and controls. Exposure to showers and toilet facilities was not shown to be a risk factor. A significant difference was found in one particular area: cases were more likely to have worked daily in the reactor and shielded facilities building.

The source of the infection appeared to be in the South Road area of the site, alongside these two buildings. A search was made for water sources producing aerosols in this area, and four cooling towers were located about 30 metres to the south. These towers served a compressor plant, which was only used intermittently, so that when not in use the residual water stagnated. Furthermore, many of the wooden drift eliminators on top of the towers, against which the steam condensed and dripped back, were defective or absent, so bacteria-laden vapour was released into the surroundings. These towers were immediately taken out of service. Samples of 25 litres of water were taken and sent to the research laboratory at Porton Down, when Legionella pneumophila was produced after guinea pig inoculation from all four towers, but not from other cooling towers on site.

\section{PUBLIC AND INDUSTRIAL RELATIONS}

At the time, with knowledge of outbreaks in Spanish hotels, Legionnaires' disease was established in peoples' minds as a new, very infectious and dangerous disease. 'Killer virus' was an eye-catching headline. Thus in the Heysham outbreak, there was an initial flurry of nationwide press cover, generally of a very responsible nature, with rather more sustained interest from the local newspaper.

Diagnosis is only confidently confirmed by very high or definitely rising titres of antibody. Because several initial results were equivocal it was in some cases not possible to tell an individual whether he had an illness caused by Legionella or just a coincidental subclinical infection. Similarly, it was not possible to give the union representatives the exact number of men involved. It further became apparent that not all medical practitioners were aware of the significance of antibody titres, so individuals sometimes received conflicting opinions.

The senior union representatives were informed as soon as the two locally hospitalised workers were diagnosed. Not having been told of the first case, there was a conviction that there had been an attempt at a 'cover-up'. When not given a guarantee that there was no risk of any further cases, the workforce walked out, returning after five days (including a weekend). There may have been other factors - it was cold, wet and schools' half-term. Added to this was apprehension about future employment prospects at a time of an inhospitable economic climate, with most of the major construction work at Phase 1 completed.

That was not the end of the story. Compensation was sought from someone or somewhere. Along with others, Dr Dyer received a writ, renewed annually for nine years, to the effect that as medical officer of health, it was his duty to have prevented the outbreak. This was so unusual that the Department of Health offered to act on his behalf. The case was eventually withdrawn on the instructions of the High Court.

This was the only occasion on which a cooling water system had been clearly implicated as a source of an outbreak of Legionellosis. In the period 1979 to 1982,32 outbreaks of Legionnaires' disease were identified in England and Wales, but this was the only one in which a cooling tower was implicated as a source of infection. In the majority of other instances domestic hot water systems in the buildings (hotels and hospitals) were found to have been the source. 